\title{
EIGENVALUE BOUNDS FOR SCHRÖDINGER OPERATORS WITH COMPLEX POTENTIALS
}

\author{
RUPERT L. FRANK
}

\begin{abstract}
We show that the absolute values of non-positive eigenvalues of Schrödinger operators with complex potentials can be bounded in terms of $L_{p}$-norms of the potential. This extends an inequality of Abramov, Aslanyan, and Davies to higher dimensions and proves a conjecture by Laptev and Safronov. Our main ingredient are the uniform Sobolev inequalities of Kenig, Ruiz, and Sogge.
\end{abstract}

Introduction and main result. Recent years have seen an increasing interest in the spectral properties of non-selfadjoint differential operators. This theory is much less developed than the self-adjoint theory, and the absence of a spectral theorem and the lack of variational techniques poses great challenges, both on a theoretical and a numerical level. We refer to [Da for an overview, physical motivations, and a wealth of (counter)examples in the field.

In this paper we will be concerned with the situation of a non-selfadjoint Schrödinger operator $-\Delta+V$ in $\mathbb{R}^{d}$ with a complex-valued potential $V$ which decays at infinity (in some averaged sense). The essential spectrum of this operator acting in $L_{2}\left(\mathbb{R}^{d}\right)$ is $[0, \infty)$, and we are interested in the location of its discrete eigenvalues. It is a beautiful observation of $\mathrm{AbAsDa}$ (see also $\mathrm{DaNa}$ ) that in dimension $d=1$, every eigenvalue $\lambda \in \mathbb{C} \backslash[0, \infty)$ of $-\frac{d^{2}}{d x^{2}}+V$ satisfies

$$
|\lambda|^{1 / 2} \leq \frac{1}{2} \int_{\mathbb{R}}|V(x)| d x .
$$

In [FrLaLiSe we extended this bound to higher dimensions, to $L_{p}$-norms of $V$ with $p \neq 1$ and to sums of eigenvalues. The prize for these generalizations is, however, that the bounds are only valid for eigenvalues outside the cone $\{z \in \mathbb{C}:|\operatorname{Im} z|<\varepsilon \operatorname{Re} z\}$ around the positive half-axis, with constants blowing up as $\varepsilon \rightarrow 0$. The recent papers [LaSa, Sa] have led to a better understanding of eigenvalues lying close to the positive half-axis. The natural generalization of (1), however,

$$
|\lambda|^{\gamma} \leq D_{\gamma, d} \int_{\mathbb{R}^{d}}|V(x)|^{\gamma+d / 2} d x
$$

for $d \geq 2$ and $0<\gamma \leq d / 2$, was left as an open conjecture in [LaSa].

Our goal in this paper is to prove this conjecture for $0<\gamma \leq 1 / 2$. The motivation in [LaSa] for the value $\gamma=d / 2$ together with the example in [IoJe] suggest that our range of $\gamma$ 's is indeed best possible.

(C) 2010 by the author. This paper may be reproduced, in its entirety, for non-commercial purposes. 
Theorem 1. Let $d \geq 2$ and $0<\gamma \leq 1 / 2$. Then any eigenvalue $\lambda \in \mathbb{C} \backslash[0, \infty)$ of the Schrödinger operator $-\Delta+V$ satisfies

$$
|\lambda|^{\gamma} \leq D_{\gamma, d} \int_{\mathbb{R}^{d}}|V(x)|^{\gamma+d / 2} d x
$$

with a constant $D_{\gamma, d}$ independent of $V$.

We also prove the formal analogue of inequality (3) for $\gamma=0$. For $d=3$ we can even evaluate the sharp value of the constant.

Theorem 2. Let $d \geq 3$. If

$$
D_{0, d} \int_{\mathbb{R}^{d}}|V(x)|^{d / 2} d x<1,
$$

then the Schrödinger operator $-\Delta+V$ has no eigenvalue in $\mathbb{C} \backslash[0, \infty)$. For $d=3$, one can take $D_{0,3}=\frac{4}{3^{3 / 2} \pi^{2}}$ and this is the smallest possible constant.

Actually, we shall prove that (3) and (44) remain true if $\int|V|^{\gamma+d / 2} d x$ is replaced by the Morrey-Campanato norm

$$
\sup _{x, r} r^{d}\left(r^{-d} \int_{B_{r}(x)}|V(y)|^{p} d y\right)^{(2 \gamma+d) / 2 p}
$$

for $p_{\gamma}<p \leq \gamma+d / 2$. This norm is weaker than the $L_{\gamma+d / 2}$-norm entering in (3) and (4) and even weaker than the weak- $L_{\gamma+d / 2}$-norm. This allows for stronger local singularities than the standard $L_{p}$-norms (e.g., for $|x|^{-2}$ singularities in the estimate analogous to (4)). The resulting eigenvalue bounds seem to be new even for real-valued potentials.

As is well-known, inequality (3) is true for real-valued potentials, even for all $\gamma>0$. This is an easy consequence of the Sobolev inequalities and the variational characterization of eigenvalues. There is no variational principle in the case of complex-valued potentials, but this impass can be circumvented using the Birman-Schwinger principle similarly as in AbAsDa. In contrast to the self-adjoint case, however, the classical Sobolev inequalities do not suffice for the proof of (3). We shall use the much deeper, 'uniform Sobolev inequalities' of Kenig, Ruiz, and Sogge [KeRuSo].

A fundamental insight of Safronov $\mathrm{Sa}$ is that methods from stationary scattering theory can be used to control eigenvalues of non-selfadjoint Schrödinger operators close to the essential spectrum. While the classical (self-adjoint) scattering theory of Agmon, Kato and Kuroda uses the Sobolev trace theorem for the restriction to the sphere of constant energy, the papers [GoSc, IoSc] advocate the use of the Stein-Tomas restriction theorem and the closely related uniform Sobolev inequalities. In the same spirit, we will replace Sobolev's restriction theorem in Sa by the uniform Sobolev inequalities in order to prove conjecture (2). 
Proofs. The proofs of both theorems are based on the Birman-Schwinger principle: if $\lambda \in \mathbb{C} \backslash[0, \infty)$ is an eigenvalue of the Schrödinger operator $-\Delta+V$, then -1 is an eigenvalue of the Birman-Schwinger operator $V^{1 / 2}(-\Delta-\lambda)^{-1}|V|^{1 / 2}$. Here we use the abbreviation $V^{1 / 2}=(\operatorname{sgn} V)|V|^{1 / 2}$. If -1 is an eigenvalue of an operator, then the norm of this operator is at least 1 . Hence Theorem 1 will follow if we can prove the bound

$$
\left\|V^{1 / 2}(-\Delta-\lambda)^{-1}|V|^{1 / 2}\right\|^{\gamma+d / 2} \leq D_{\gamma, d}|\lambda|^{-\gamma} \int_{\mathbb{R}^{d}}|V(x)|^{\gamma+d / 2} d x .
$$

Similarly, Theorem 2 will follow if we can prove the bound

$$
\left\|V^{1 / 2}(-\Delta+\lambda)^{-1}|V|^{1 / 2}\right\|^{d / 2} \leq D_{0, d} \int_{\mathbb{R}^{d}}|V(x)|^{d / 2} d x .
$$

As a warm-up, we begin with bound (6) for $d=3$. In this case, the integral kernel of $(-\Delta-\lambda)^{-1}$ is given by $(4 \pi|x-y|)^{-1} e^{i \sqrt{\lambda}|x-y|}$, where we use the branch of the square root on $\mathbb{C} \backslash[0, \infty)$ with positive imaginary part. Hence this kernel is bounded in absolute value by $(4 \pi|x-y|)^{-1}$, the Green's function. We conclude that for any $\varphi, \psi \in L_{2}\left(\mathbb{R}^{3}\right)$,

$$
\begin{aligned}
\left|\left(\varphi, V^{1 / 2}(-\Delta-\lambda)^{-1}|V|^{1 / 2} \psi\right)\right| & \leq \frac{1}{4 \pi} \iint \frac{|\varphi(x)||V(x)|^{1 / 2}|V(y)|^{1 / 2}|\psi(y)|}{|x-y|} d x d y \\
& \leq \frac{2^{4 / 3}}{3 \pi^{4 / 3}}\left\|\varphi|V|^{1 / 2}\right\|_{6 / 5}\left\|\psi|V|^{1 / 2}\right\|_{6 / 5},
\end{aligned}
$$

where in the last step we applied Lieb's sharp version of the Hardy-Littlewood-Sobolev inequality [Li] (see also [LiLo]). Finally, we bound using Hölder's inequality

$$
\left\|\psi|V|^{1 / 2}\right\|_{6 / 5} \leq\|V\|_{3 / 2}^{1 / 2}\|\psi\|_{2},
$$

and similarly for $\left\|\varphi|V|^{1 / 2}\right\|_{6 / 5}$. Taking the supremum over all $\psi$ and $\varphi$ with $L_{2}$-norm equal to one, we conclude that

$$
\left\|V^{1 / 2}(-\Delta+\lambda)^{-1}|V|^{1 / 2}\right\| \leq \frac{2^{4 / 3}}{3 \pi^{4 / 3}}\|V\|_{3 / 2} .
$$

This proves the assertion of Theorem 2 for $d=3$ with the claimed constant.

This constant is sharp, even if the inequality is restricted to real-valued potentials. Indeed, the potential $V(x)=-3\left(1+|x|^{2}\right)^{-2}$ leads to a zero-energy resonance (with resonance function $\left.\psi(x)=\left(1+|x|^{2}\right)^{-1 / 2}\right)$, and any negative perturbation turns this resonance into a negative eigenvalue. Moreover, for this potential $\int|V|^{3 / 2} d x=3^{3 / 2} \pi^{2} / 4$.

We now turn to the general case. As we already mentioned, the key ingredient is the uniform Sobolev inequality by Kenig, Ruiz and Sogge [KeRuSo], which asserts that

$$
\left\|(-\Delta-\lambda)^{-1}\right\|_{L_{p} \rightarrow L_{p^{\prime}}} \leq C_{p, d}|\lambda|^{-\frac{d+2}{2}+\frac{d}{p}}
$$

for $\frac{2 d}{d+2} \leq p \leq \frac{2(d+1)}{d+3}$ if $d \geq 3$ and for $1<p \leq \frac{6}{5}$ if $d=2$. Here, as usual, $\frac{1}{p}+\frac{1}{p^{\prime}}=1$. To be more precise, the claimed $|\lambda|$-decay in (7) is not stated in KeRuSo, but once the inequality is established uniformly for $|\lambda|=1, \lambda \neq 1$, the decay follows easily 
by scaling. Moreover, in [KeRuSo] the inequality is only proved for $d \geq 3$, but their argument extends to the two-dimensional case provided $p=1$ is excluded. (For $d=2$ one omits the gamma function factor in [KeRuSo, (2.17)] and verifies [KeRuSo, (2.20)] for $\operatorname{Re}(\lambda) \in[-3 / 2,1)$.)

With (7) at hand we can estimate similarly as before

$$
\begin{aligned}
\left|\left(\varphi, V^{1 / 2}(-\Delta+\lambda)^{-1}|V|^{1 / 2} \psi\right)\right| & \leq C_{p, d}|\lambda|^{-\frac{d+2}{2}+\frac{d}{p}}\left\|\varphi|V|^{1 / 2}\right\|\left\|_{p}\right\| \psi|V|^{1 / 2} \|_{p} \\
& \leq C_{p, d}|\lambda|^{-\frac{d+2}{2}+\frac{d}{p}}\|V\|_{p /(2-p)}\|\varphi\|_{2}\|\psi\|_{2} .
\end{aligned}
$$

For given $0<\lambda \leq 1 / 2$ (as well as $\lambda=0$ for $d \geq 3$ ) we are allowed to choose $p=2(2 \gamma+d) /(2 \gamma+d+2)$. Taking again the supremum over all $\psi$ and $\varphi$ with $L_{2^{-}}$ norm equal to one we arrive at (5) and (6) with $D_{\gamma, d}=C_{p, d}^{\gamma+d / 2}$. This completes the proofs of Theorems 1 and 2 .

Extension to singular potentials. We prove eigenvalue inequalities for potentials with stronger (local) singularities than those allowed in Theorems 1 and 2. For that purpose we measure the size of a potential by its norm in the Morrey-Campanato space $\mathcal{L}^{\alpha, p}\left(\mathbb{R}^{d}\right)$

$$
\|V\|_{\mathcal{L}^{\alpha, p}}:=\sup _{x, r} r^{\alpha}\left(r^{-d} \int_{B_{r}(x)}|V(y)|^{p} d y\right)^{1 / p} .
$$

One easily shows the (continuous) inclusions

$$
\mathcal{L}^{\alpha, p}\left(\mathbb{R}^{d}\right) \supset \mathcal{L}^{\alpha, r}\left(\mathbb{R}^{d}\right) \supset L_{d / \alpha, \infty}\left(\mathbb{R}^{d}\right) \supset \mathcal{L}^{\alpha, d / \alpha}\left(\mathbb{R}^{d}\right)=L_{d / \alpha}\left(\mathbb{R}^{d}\right) \quad \text { for } 1 \leq p<r<d / \alpha .
$$

(Indeed, one can prove that these inclusions are all strict.) We shall prove

Theorem 3. Let $d \geq 2,0<\gamma<1 / 2$ and $(d-1)(2 \gamma+d) / 2(d-2 \gamma)<p \leq \gamma+d / 2$. Then any eigenvalue $\lambda \in \mathbb{C} \backslash[0, \infty)$ of the Schrödinger operator $-\Delta+V$ satisfies

$$
|\lambda|^{\gamma} \leq D_{\gamma, d, p} \sup _{x, r} r^{d}\left(r^{-d} \int_{B_{r}(x)}|V(y)|^{p} d y\right)^{(2 \gamma+d) / 2 p} .
$$

Moreover, if $d \geq 3,(d-1) / 2<p \leq d / 2$ and

$$
D_{0, d, p} \sup _{x, r} r^{d}\left(r^{-d} \int_{B_{r}(x)}|V(y)|^{p} d y\right)^{d / 2 p}<1,
$$

then the Schrödinger operator $-\Delta+V$ has no eigenvalue in $\mathbb{C} \backslash[0, \infty)$.

For the proof we replace (7) by a weighted $L_{2}$-estimate. The $L_{2}$-space on $\mathbb{R}^{d}$ with measure $\omega(x) d x$ is denoted by $L_{2}(\omega)$.

Lemma 4. Let $4 / 3<\alpha<2$ if $d=2,2 d /(d+1)<\alpha \leq 2$ if $d \geq 3$ and let $(d-1) / 2(\alpha-1)<p \leq d / \alpha$. Then for all $0<\omega \in \mathcal{L}^{\alpha, p}\left(\mathbb{R}^{d}\right)$,

$$
\left\|(-\Delta-\lambda)^{-1}\right\|_{L_{2}\left(\omega^{-1}\right) \rightarrow L_{2}(\omega)} \leq C_{d, \alpha, p}\|\omega\|_{\mathcal{L}^{\alpha, p}}|\lambda|^{-1+\alpha / 2} .
$$


This bound is essentially contained in the works [ChSa, $\mathrm{ChRu}$, but not in this explicit form and only for $d \geq 3$. Therefore we provide a short sketch of a proof in the final section below. Assuming it for the moment, we choose a strictly positive function $g \in \mathcal{L}^{\alpha, p}$ (a Gaussian, say) and define $V_{\varepsilon}(x):=\sup \{|V(x)|, \varepsilon g(x)\}$. Then by Lemma 4

$$
\begin{aligned}
\left|\left(\varphi, V^{1 / 2}(-\Delta+\lambda)^{-1}|V|^{1 / 2} \psi\right)\right| & \leq C_{d, \alpha, p}|\lambda|^{-1+\alpha / 2}\left\|V_{\varepsilon}\right\|_{\mathcal{L}^{\alpha, p}}\left\|\sqrt{|V| / V_{\varepsilon}} \varphi\right\|_{2}\left\|\sqrt{|V| / V_{\varepsilon}} \psi\right\|_{2} \\
& \leq C_{d, \alpha, p}|\lambda|^{-1+\alpha / 2}\left\|V_{\varepsilon}\right\|_{\mathcal{L}^{\alpha, p}}\|\varphi\|_{2}\|\psi\|_{2} .
\end{aligned}
$$

Hence, as $\varepsilon \rightarrow 0$,

$$
\left\|V^{1 / 2}(-\Delta+\lambda)^{-1}|V|^{1 / 2}\right\| \leq C_{d, \alpha, p}|\lambda|^{-1+\alpha / 2}\|V\|_{\mathcal{L}^{\alpha, p}}
$$

which is the analogue of (5) and (6). Theorem 3 follows by choosing $\alpha=2 d /(2 \gamma+d)$.

Extension to slowly decaying potentials. In our previous theorems, we essentially required the potential to decay like $|x|^{-\rho}$ with $\rho>2 d /(d+1)$. In order to deal with the case $\rho>1$, we recall the following weighted norm estimate for the resolvent $(-\Delta-\lambda)^{-1}$, which plays a crucial role in stationary scattering theory,

$$
\left\|(-\Delta-\lambda)^{-1}\right\|_{L_{2}\left(\left(1+|x|^{2}\right)^{\alpha}\right) \rightarrow L_{2}\left(\left(1+|x|^{2}\right)^{-\alpha}\right)} \leq C_{d, \alpha}|\lambda|^{-1 / 2} \quad \text { if } \alpha>1 / 2 .
$$

The dependence on $\lambda$ can be improved to $(1+|\lambda|)^{-1 / 2}$, but (12) is enough for our purposes. We refer to [Sy] for the $\alpha$ dependence. In contrast to (7) and (11), $|\lambda|$ enters (12) with the (optimal) power $-1 / 2$. Improvements in this endpoint case can be found in BaRuVe.

The same argument as in the previous subsection, but with $V_{\varepsilon}$ replaced by $\left(1+|x|^{2}\right)^{\alpha}$, leads to the bound

$$
|\lambda|^{1 / 2} \leq C_{d, \alpha} \sup _{x}\left(1+|x|^{2}\right)^{\alpha}|V(x)|, \quad \alpha>1 / 2
$$

thus rederiving the result from $[\mathrm{Sa}]$. By interpolating this with Theorem 1 we obtain

Theorem 5. Let $d \geq 2, \gamma>1 / 2$ and $\alpha>\gamma-1 / 2$. Then any non-positive eigenvalue $\lambda \in \mathbb{C} \backslash[0, \infty)$ of the Schrödinger operator $-\Delta+V$ satisfies

$$
|\lambda|^{\gamma} \leq C_{d, \gamma, \alpha} \int_{\mathbb{R}^{d}}|V(x)|^{2 \gamma+(d-1) / 2}\left(1+|x|^{2}\right)^{\alpha} d x .
$$

Indeed, interpolation between (7) with $p_{d}:=2(d+1) /(d+3)$ and (12) yields

$$
\left\|\left(1+|x|^{2}\right)^{-\alpha \theta / 2}(-\Delta-\lambda)^{-1}\left(1+|x|^{2}\right)^{-\alpha \theta / 2}\right\|_{L_{p} \rightarrow L_{p^{\prime}}} \leq C_{d, \alpha, p}|\lambda|^{-\frac{1-\theta}{d+1}-\frac{\theta}{2}}, \quad \frac{1}{p}=\frac{1-\theta}{p_{d}}+\frac{\theta}{2},
$$

for $\alpha>1 / 2$, and then the argument of the previous subsection implies (13). 
Proof of Lemma 4. As a preliminary step we deduce from the works [ChWh, SaWh that the operator $I_{\alpha}$ of convolution with $|x|^{-d+\alpha}, 0<\alpha<d$, satisfies

$$
\left\|I_{\alpha}\right\|_{L_{2}\left(w^{-1}\right) \rightarrow L_{2}(w)} \leq C_{d, \alpha, s}\|w\|_{\mathcal{L}^{\alpha, s}}
$$

for any $1<s \leq d / \alpha$. Indeed, the constant $C_{r}$ in [SaWh, (1.10)] for $w=v^{-1}$ and $p=2$ is bounded by a constant times $\|w\|_{\mathcal{L}^{\alpha, r}}$, and this constant enters multiplicatively in [SaWh, (2.9)].

After this preliminary step we turn to the proof of estimate (11). Following [KeRuSo, ChSa we consider the analytic family of operators $\omega^{\zeta / 2}(-\Delta-\lambda)^{-\zeta} \omega^{\zeta / 2}$ with $0 \leq \operatorname{Re} \zeta \leq$ $(d+1) / 2$. The bounds in [KeRuSo] for the (explicit) integral kernel of $(-\Delta-\lambda)^{-\zeta}$ show that

$$
\left|(-\Delta-\lambda)^{-\zeta}\left(x, x^{\prime}\right)\right| \leq C_{d} e^{C|\operatorname{Im} \zeta|}\left|x-x^{\prime}\right|^{-(d+1) / 2+\operatorname{Re} \zeta}
$$

for $(d-1) / 2 \leq \operatorname{Re} \zeta \leq(d+1) / 2$ with $C$ independent of $\lambda$ satisfying $|\lambda|=1$. This, together with (14), proves that

$$
\begin{aligned}
\left\|\omega^{\zeta / 2}(-\Delta-\lambda)^{-\zeta} \omega^{\zeta / 2}\right\|_{L_{2} \rightarrow L_{2}} & \leq C e^{C|\operatorname{Im} \zeta|}\left\|\omega^{\operatorname{Re} \zeta}\right\|_{\mathcal{L}^{\operatorname{Re} \zeta+(d-1) / 2, s}} \\
& =C e^{C|\operatorname{Im} \zeta|}\|\omega\|_{\mathcal{L}^{1+(d-1) /(2 \operatorname{Re} \zeta), s \operatorname{Re} \zeta}}^{\operatorname{Re} \zeta}
\end{aligned}
$$

for $s>1$. Complex interpolation with the trivial bound for $\operatorname{Re} \zeta=0$ yields

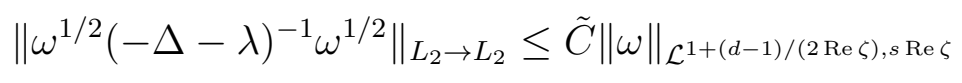

for $(d-1) / 2 \leq \operatorname{Re} \zeta \leq(d+1) / 2$ if $d \geq 3$ and for $1 \leq \operatorname{Re} \zeta \leq 3 / 2$. This is the claimed bound for $|\lambda|=1$, and the case of general $\lambda$ follows by scaling. (Note that $2 d /(d+1) \leq \alpha=1+(d-1) /(2 \operatorname{Re} \zeta) \leq 2$ corresponds to $(d-1) / 2 \leq \operatorname{Re} \zeta \leq(d+1) / 2$.

It remains to prove the case $\alpha \in(3 / 2,2)$ for $d=2$. A similar (but simpler) analysis as in [KeRuSo shows that for $d=2$ and any $3 / 2<\alpha<2$ one has

$$
\left|(-\Delta-\lambda)^{-1}\left(x, x^{\prime}\right)\right| \leq C_{\alpha}\left|x-x^{\prime}\right|^{-2+\alpha}
$$

uniformly in $|\lambda|=1$. This, together with (14), completes the proof of Lemma 4.

The author wishes to thank A. Laptev and O. Safronov for useful correspondence.

\section{REFERENCES}

[AbAsDa] A. A. Abramov, A. Aslanyan, E. B. Davies, Bounds on complex eigenvalues and resonances. J. Phys. A 34 (2001), no. 1, 57-72.

[BaRuVe] J. A. Barcelo, A. Ruiz, L. Vega, Weighted estimates for the Helmholtz equation and some applications. J. Funct. Anal. 150 (1997), no. 2, 356-382.

[ChSa] S. Chanillo, E. Sawyer, Unique continuation for $\Delta+v$ and the C. Fefferman-Phong class. Trans. Amer. Math. Soc. 318 (1990), no. 1, 275-300.

[ChWh] S. Chanillo, R. L. Wheeden, $L^{p}$-estimates for fractional integrals and Sobolev inequalities with applications to Schrödinger operators. Comm. Partial Differential Equations 10 (1985), no. 9, 1077-1116.

[ChRu] F. Chiarenza, A. Ruiz, Uniform $L^{2}$-weighted Sobolev inequalities. Proc. Amer. Math. Soc. 112 (1991), no. 1, 53-64. 
[Da] E. B. Davies, Non-self-adjoint differential operators. Bull. London Math. Soc. 34 (2002), no. $5,513-532$.

[DaNa] E. B. Davies, J. Nath, Schrödinger operators with slowly decaying potentials. J. Comput. Appl. Math. 148 (2002), no. 1, 1-28.

[GoSc] M. Goldberg, W. Schlag, A limiting absorption principle for the three-dimensional Schrödinger equation with $L^{p}$ potentials. Int. Math. Res. Not. 2004, no. 75, 4049-4071.

[FrLaLiSe] R. L. Frank, A. Laptev, E. H. Lieb, R. Seiringer, Lieb-Thirring inequalities for Schrödinger operators with complex-valued potentials. Lett. Math. Phys. 77 (2006), no. 3, 309-316.

[IoSc] A. D. Ionescu, W. Schlag, Agmon-Kato-Kuroda theorems for a large class of perturbations. Duke Math. J. 131 (2006), no. 3, 397-440.

[IoJe] A. D. Ionescu, D. Jerison, On the absence of positive eigenvalues of Schrödinger operators with rough potentials. Geom. Funct. Anal. 13 (2003), 1029-1081.

[KeRuSo] C. E. Kenig, A. Ruiz, C. D. Sogge, Uniform Sobolev inequalities and unique continuation for second order constant coefficient differential operators. Duke Math. J. 55 (1987), no. 2, 329-347.

[LaSa] A. Laptev, O. Safronov, Eigenvalue estimates for Schrödinger operators with complex potentials. Comm. Math. Phys. 292 (2009), no. 1, 29-54.

[Li] E. H. Lieb, Sharp constants in the Hardy-Littlewood-Sobolev and related inequalities. Ann. of Math. (2) 118 (1983), no. 2, 349-374.

[LiLo] E. H. Lieb, M. Loss, Analysis. Second edition. Graduate Studies in Mathematics 14, American Mathematical Society, Providence, RI, 2001.

[Sa] O. Safronov, Estimates for eigenvalues of the Schrödinger operator with a complex potential. Preprint (2009), arXiv:0902.3950.

[SaWh] E. Sawyer, R. L. Wheeden, Weighted inequalities for fractional integrals on Euclidean and homogeneous spaces. Amer. J. Math. 114 (1992), no. 4, 813-874.

[Sy] J. Sylvester, An estimate for the free Helmholtz equation that scales. Inverse Probl. Imaging 3 (2009), no. 2, 333-351.

Rupert L. Frank, Department of Mathematics, Princeton University, Princeton, NJ 08544, USA

E-mail address: rlfrank@math.princeton.edu 